\title{
Frequency and clinical outcomes of CYP2C19 genotype-guided escalation and de-escalation of antiplatelet therapy in a real-world clinical setting
}

\author{
Jesse Martin, BS ${ }^{1}{ }^{1}$, Alexis K. Williams, PharmD (i) ${ }^{1}$, Melissa D. Klein, BS², Vindhya B. Sriramoju, MD², \\ Shivanshu Madan, MD², Joseph S. Rossi, MD², Megan Clarke, PharmD³, Jonathan D. Cicci, PharmD (iD ${ }^{3}$, \\ Larisa H. Cavallari, PharmD ${ }^{4}$, Karen E. Weck, MD ${ }^{5}{ }^{5}$, George A. Stouffer, MD $\mathbb{D}^{2,6}$ and \\ Craig R. Lee, PharmD, PhD ${ }^{1,6}$
}

Purpose: To evaluate the frequency and clinical impact of switches in antiplatelet therapy following implementation of CYP2C19 genotyping after percutaneous coronary intervention (PCI).

Methods: The frequency of escalation (clopidogrel switched to prasugrel/ticagrelor) and de-escalation (prasugrel/ticagrelor switched to clopidogrel) was evaluated in 1063 PCI patients who underwent CYP2C19 genotyping. Risk of major adverse cardiovascular or cerebrovascular (MACCE) and bleeding events over one year was evaluated.

Results: Antiplatelet therapy switches were common (19\%), with escalation (101/115: 88\%) and de-escalation (77/84: 92\%) occurring predominantly in patients with and without a CYP2C19 nonfunctional allele, respectively. Nonfunctional allele carriers initiated and continued on clopidogrel had a significantly higher risk of experiencing either a MACCE or bleeding event compared with those escalated to prasugrel/ticagrelor (52 vs. 19 events/100 patient- years; adjusted hazard ratio [HR] 2.89 [1.44-6.13], $p=0.003)$. Patients without a nonfunctional allele de-escalated to clopidogrel had no difference in risk compared with those initiated and continued on prasugrel/ticagrelor (21 vs. 19 events/100 patientyears; adjusted HR 1.13 [0.51-2.34], $p=0.751$ ).

Conclusion: CYP2C19-guided escalation and de-escalation is common in a real-world setting. Continuation of clopidogrel in nonfunctional allele carriers is associated with adverse outcomes. De-escalation to clopidogrel in patients without a nonfunctional allele appears safe and warrants prospective study.

Genetics in Medicine (2020) 22:160-169; https://doi.org/10.1038/s41436019-0611-1

Keywords: Antiplatelet drug; switching; clopidogrel; cytochrome P450 enzymes; pharmacogenetics

\section{INTRODUCTION}

Over 480,000 percutaneous coronary interventions (PCIs) are performed annually in the United States. ${ }^{1}$ Dual antiplatelet therapy with aspirin and a $\mathrm{P}_{2} \mathrm{Y}_{12}$ inhibitor (clopidogrel, prasugrel, or ticagrelor) is indicated after PCI for 1 year. $^{2}$ Clopidogrel is a prodrug that requires bioactivation by the CYP2C19 enzyme; however, CYP2C19 nonfunctional ( ${ }^{*} 2$ and ${ }^{\star} 3$ ) polymorphisms are common and significantly impair clopidogrel active metabolite formation, platelet inhibition, and clinical effectiveness. ${ }^{3,4}$ In contrast, prasugrel and ticagrelor exhibit more potent and consistent antiplatelet effects, and superior efficacy in acute coronary syndrome (ACS) patients, compared with clopidogrel. ${ }^{5,6}$ Moreover, CYP2C19 genotype does not impact the clinical response to prasugrel or ticagrelor. ${ }^{7,8}$ However, these alternative therapies have higher bleeding risk, discontinuation rates, and cost compared with clopidogrel. ${ }^{5,}$

In clinical practice, switching between $\mathrm{P}_{2} \mathrm{Y}_{12}$ inhibitors has become increasingly common and is driven by clinical and socioeconomic factors. ${ }^{11,12}$ Understanding the frequency and reasons for switching from clopidogrel to prasugrel or ticagrelor (termed "escalation") or from prasugrel or ticagrelor to clopidogrel (termed "de-escalation") has emerged as a major area of investigation. ${ }^{13-18}$ Notably, accumulating data demonstrate that use of genotyping to guide escalation from clopidogrel to prasugrel/ticagrelor in CYP2C19 nonfunctional allele carriers after PCI lowers the risk of atherothrombotic events compared with conventional treatment strategies without significantly increasing bleeding risk. ${ }^{19-21}$ Multiple institutions

${ }^{1}$ Division of Pharmacotherapy and Experimental Therapeutics, UNC Eshelman School of Pharmacy, University of North Carolina at Chapel Hill, Chapel Hill, NC, USA; ${ }^{2}$ Division of Cardiology, UNC School of Medicine, University of North Carolina at Chapel Hill, Chapel Hill, NC, USA; ${ }^{3}$ Department of Pharmacy, UNC Medical Center, Chapel Hill, NC, USA; ${ }^{4}$ Department of Pharmacotherapy and Translational Research and Center for Pharmacogenomics, University of Florida, Gainesville, FL, USA; ${ }^{5}$ Department of Pathology and Laboratory Medicine, UNC School of Medicine, University of North Carolina at Chapel Hill, Chapel Hill, NC, USA; ${ }^{6}$ UNC McAllister Heart Institute, University of North Carolina at Chapel Hill, Chapel Hill, NC, USA. Correspondence: Craig R. Lee (craig_lee@unc.edu) 
have successfully implemented CYP2C19-guided antiplatelet therapy into clinical workflows. ${ }^{22}$ Given that genotype tests are typically ordered reactively, with results obtained after PCI, CYP2C19-guided selection of antiplatelet therapy inherently requires switching between $\mathrm{P} 2 \mathrm{Y}_{12}$ inhibitors. ${ }^{22}$

Clinical practice guidelines now recommend preferential use of prasugrel or ticagrelor in ACS patients undergoing $\mathrm{PCI},{ }^{2}$ though clopidogrel remains the most widely prescribed $\mathrm{P}_{2} \mathrm{Y}_{12}$ inhibitor and the only agent indicated for PCI in the setting of stable disease. ${ }^{9}$ As a consequence, use of prasugrel or ticagrelor early after PCI, when the risk for ischemic events is highest, followed by de-escalation to clopidogrel maintenance therapy to reduce bleeding risk and lower medication costs has become more common in practice; ${ }^{11,12,23}$ however, the safety and effectiveness of empiric de-escalation remain unclear and studies have yielded conflicting results. ${ }^{16,24}$ Biomarker-guided strategies offer the potential to more precisely direct de-escalation and improve clinical outcomes. ${ }^{25}$ Thus, use of CYP2C19 genotyping to guide deescalation from prasugrel/ticagrelor to clopidogrel in patients without a nonfunctional allele has emerged as a new model in genotype-guided antiplatelet therapy.

The frequency and timing of CYP2C19 genotype-guided deescalation of antiplatelet therapy in a real-world setting have not been evaluated, and the impact on clinical outcomes is unknown. The study objectives were to (1) describe the frequency and timing of switches between $\mathrm{P}_{2} \mathrm{Y}_{12}$ inhibitors following implementation of a genotype-guided treatment algorithm, (2) evaluate the impact of CYP2C19 results on $\mathrm{P}_{2} \mathrm{Y}_{12}$ inhibitor escalation and de-escalation, and (3) examine the relationship between escalation and de-escalation, CYP2C19 status, and clinical outcomes post-PCI.

\section{MATERIALS AND METHODS}

\section{Study design and population}

A clinical algorithm for CYP2C19 genotype-guided antiplatelet selection following PCI in high-risk patients (defined as ACS or high-risk coronary anatomy) was implemented at the University of North Carolina-Chapel Hill (UNC) as described. $^{26,27}$ This single-center observational cohort study included 1493 consecutive patients $\geq 18$ years of age who underwent coronary artery stent placement at UNC Medical Center from 1 July 2012 to 31 December 2014 and received $\mathrm{P}_{2} \mathrm{Y}_{12}$ inhibitor treatment. The investigation was approved by the UNC Biomedical Institutional Review Board. Because data was retrospectively abstracted from the electronic health record (EHR), informed consent was not required.

Initial antiplatelet therapy was selected based on clinical factors, and CYP2C19 genotype tests were ordered at the interventional cardiologist's discretion. Clinical testing for the CYP $2 C 19^{\star} 2{ }^{*} 3$, and ${ }^{*} 17$ alleles was performed by the UNC Molecular Genetics Laboratory, with return of results in the EHR, as described. ${ }^{26,27}$ Metabolizer phenotypes were assigned according to Clinical Pharmacogenetics Implementation Consortium (CPIC) recommendations: ultrarapid (UM; ${ }^{\star} 17 /$ $\left.{ }^{\star} 17\right)$, rapid $\left(\mathrm{RM} ;{ }^{\star} 1 /{ }^{\star} 17\right)$, normal $\left(\mathrm{NM} ;{ }^{\star} 1 /{ }^{\star} 1\right)$, intermediate

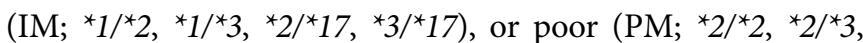
$\left.\star 3 /{ }^{*} 3\right) .{ }^{28}$ Prasugrel or ticagrelor was recommended in nonfunctional allele carriers (IMs and PMs); however, the decision to continue the initial agent or switch agents, and the time to switch after PCI, was left to prescriber discretion (Fig. S1).

\section{Data abstraction and study endpoints}

Clinical and medication data, and CYP2C19 genotype results, were abstracted from the EHR, as described. ${ }^{26}$ Initial therapy was defined as the $\mathrm{P}_{2} \mathrm{Y}_{12}$ inhibitor administered (loaded) during the PCI procedure. Maintenance therapy was the agent prescribed over the course of follow-up after any switches. A switch, which included escalation (clopidogrel switched to prasugrel/ticagrelor) or de-escalation (prasugrel/ticagrelor switched to clopidogrel), was defined as a change occurring after administration of the initial agent. Time to switch was calculated as the number of days between the PCI procedure and the date of escalation or de-escalation. Elevated risk for bleeding was a composite variable defined as the presence of one or more risk factors for bleeding on antiplatelet therapy: age $\geq 75$ years, weight $<60 \mathrm{~kg}$, previous transient ischemic attack (TIA) or cerebrovascular accident (CVA), history of a clinically significant bleeding event, end-stage renal disease requiring dialysis, or anticoagulant prescribed at discharge.

The primary clinical outcome was the composite of either a major adverse cardiovascular or cerebrovascular event (MACCE) or clinically significant bleeding event, similar to clinical trials of precision antiplatelet therapy. ${ }^{25,29}$ MACCE and clinically significant bleeding events were also analyzed individually as secondary outcomes. MACCE was defined as the composite of death, myocardial infarction (MI), stent thrombosis, hospitalization for unstable angina, ischemic stroke, or TIA, as described. ${ }^{26}$ Clinically significant bleeding was defined as Global Use of Strategies to Open Occluded Arteries (GUSTO) moderate (requiring blood transfusion but not resulting in hemodynamic compromise) or severe/lifethreatening (intracerebral hemorrhage or bleeding resulting in hemodynamic compromise requiring treatment) bleeding. ${ }^{30}$ Events were identified using physician-reported diagnoses abstracted from the EHR, and verified by an interventional cardiologist. Maintenance therapy at the time of event was recorded.

\section{Statistical analysis}

Data are presented as mean \pm standard deviation, median [interquartile range], or count (\%). $\mathrm{P}_{2} \mathrm{Y}_{12}$ inhibitor (clopidogrel or prasugrel/ticagrelor), CYP2C19 phenotype (IM/PM or $\mathrm{UM} / \mathrm{RM} / \mathrm{NM}$ ) and indication for PCI (ACS or non-ACS/ elective) were dichotomized, and demographic and clinical factors were compared across groups using Student's $t$ test, chi-square, or Fisher's exact test. Time-to-switch data were not normally distributed, and compared across groups by Wilcoxon signed rank test. Associations between CYP2C19 status and PCI indication with switches in therapy were evaluated by logistic regression. 
The time to occurrence of a clinical event within 12 months after PCI (baseline) was calculated in each patient who underwent CYP2C19 testing with follow-up information available in the EHR after the index PCI admission $(n=$ 928). Patients who did not present to UNC for outpatient or emergent care after discharge were considered lost to followup. Patients who did not experience an event were censored at the time of last encounter in which treatment with a $\mathrm{P}_{2} \mathrm{Y}_{12}$ inhibitor was documented. Event rates were reported as the number of events per 100 patient-years of follow-up.

The relationship between $\mathrm{P}_{2} \mathrm{Y}_{12}$ inhibitor maintenance therapy, CYP2C19 status, and time to occurrence of the primary and secondary clinical outcomes was evaluated by Cox proportional hazards regression, as previously described. ${ }^{19,26}$ Due to the observational study design, analyses were completed after adjusting for baseline covariates that associated with clinical outcome or differed across CYP2C19 phenotype-antiplatelet groups. Multivariable models for MACCE and bleeding were created using stepwise selection of candidate covariates, described in Table S1, with the criterion of $P<0.20$ to enter and stay in the model. Demographic factors (age, gender, African American race) and clinical factors known to influence antiplatelet therapy selection (ACS indication for PCI, elevated risk for bleeding) ${ }^{26}$ were forced into each model. Covariates in adjusted model for MACCE were age (continuous), gender, African American race, ACS indication for PCI, elevated risk of bleeding (composite), prior stent, diabetes, peripheral vascular disease, atrial fibrillation, current smoker, drug-eluting stent at index PCI, and discharge statin. Covariates in adjusted model for bleeding were age (continuous), gender, African American race, elevated risk of bleeding (composite), ACS indication for PCI, prior stent, drug-eluting stent at index PCI, and multiple vessels stented. The adjusted model for the composite MACCE or bleeding outcome included each covariate from the individual MACCE and bleeding models.

To examine the impact of genotype-guided escalation and deescalation, associations between CYP2C19 phenotype-antiplatelet groups (IM/PM-clopidogrel, IM/PM-prasugrel/ticagrelor, $\mathrm{UM} / \mathrm{RM} / \mathrm{NM}$-clopidogrel, UM/RM/NM-prasugrel/ticagrelor) and outcome were evaluated by Cox proportional hazards regression following stratification by initial antiplatelet therapy (clopidogrel: $n=612$; alternative: $n=316$ ). Covariate-adjusted hazard ratio (HR) and 95\% confidence intervals (CIs) for each between-group comparison were calculated. Secondary analyses were completed in the strata of patients presenting with an ACS indication for PCI. Kaplan-Meier curves were generated using Prism 7.0 (GraphPad Software, La Jolla. CA). Analyses were performed using SAS-JMP 12.0 and SAS 9.4 (SAS Institute, Cary, NC). $P$ values $<0.05$ were considered statistically significant.

A sample size of $n=600$ initiated on clopidogrel was estimated to provide $80 \%$ power to detect a HR $\geq 2.54(\mathrm{a}=$ 0.05 , two-sided) when comparing IM/PMs continued on clopidogrel versus IM/PMs escalated to alternative therapy (nQuery 8.2, Boston, MA), assuming 30\% IM/PM prevalence,
$50 \%$ of IM/PMs escalated to prasugrel/ticagrelor $(n=90 /$ group), and a $20 \%$ event rate in IM/PMs.

\section{RESULTS}

\section{Study population}

The mean age was $63 \pm 12$ years, $67.5 \%$ were male, and $20.1 \%$ were African American. Comorbidities such as hypertension (84.3\%), diabetes $(42.5 \%)$, and atrial fibrillation (9.9\%) were common. Overall, 54.3\% underwent PCI for ACS, and 39.2\% exhibited a risk factor for bleeding. Baseline characteristics are summarized in Table S1.

Clopidogrel (71.4\%) was the most commonly prescribed initial $\mathrm{P}_{2} \mathrm{Y}_{12}$ inhibitor, followed by prasugrel $(27.5 \%)$ and ticagrelor (1.1\%). Numerous clinical factors differed across initial therapy (Table S1). Most notably, a significantly greater proportion initiated on prasugrel/ticagrelor had an ACS indication compared with clopidogrel (70.5\% vs. $47.8 \%$, respectively, $P<0.001$ ), whereas significantly fewer initiated on prasugrel/ticagrelor exhibited elevated bleeding risk compared with clopidogrel $(17.1 \%$ vs. $48.0 \%$, respectively, $P<0.001)$.

\section{Genotype testing and maintenance therapy selection} CYP2C19 genotype was obtained in 1063 (71.2\%) of PCI patients, with results available, on average, 1 day after PCI (Table S2). A genotype was more frequently obtained in patients with an ACS compared with a non-ACS/elective indication for PCI $(78.3 \%$ vs. $62.8 \%$, respectively, $p<0.001)$. Among genotyped patients, 329 (31.0\%) carried either one (IM: 303, 28.5\%) or two (PM: 26, 2.4\%) nonfunctional alleles, and $450(42.3 \%), 239(22.5 \%)$, and $45(4.2 \%)$ were classified as a CYP2C19 NM, RM, and UM, respectively. The ${ }^{\star} 2,{ }^{\star} 3$, and ${ }^{\star} 17$ allele frequencies were $0.166,0.001$, and 0.183 , respectively, and did not deviate from Hardy-Weinberg equilibrium $(P>0.05$ for each allele).

Overall, clopidogrel (62.2\%) was the most commonly prescribed maintenance therapy. CYP2C19 IM/PMs were more frequently prescribed prasugrel/ticagrelor than $\mathrm{UM} / \mathrm{RM} / \mathrm{NMs}(68.7 \%$ vs. $24.0 \%, p<0.001) ; 67.3 \%$ of IMs (204 of 303 ) and $84.6 \%$ (22 of 26) of PMs received alternative therapy. Frequency of alternative therapy use in IM/PMs was significantly higher in ACS compared with non-ACS/elective patients $(75.5 \%$ vs. $59.1 \%, p=0.002)$ (Table S2).

\section{Changes in $\mathrm{P}^{2} \mathrm{Y}_{12}$ inhibitor therapy}

$\mathrm{P} 2 \mathrm{Y}_{12}$ inhibitor escalation or de-escalation was observed in $209(14.0 \%)$ of all PCI patients. Switches in therapy were significantly more frequent in patients with (18.7\%) vs. without $(2.3 \%)$ an available CYP2C19 genotype (OR 9.7, 95\% CI $5.1-18.5 ; p<0.001)$. In genotyped patients, both escalation from clopidogrel to prasugrel/ticagrelor (10.8\%) and deescalation from prasugrel/ticagrelor to clopidogrel (7.9\%) were common (Fig. 1a). Switches in therapy were also more frequent in ACS compared with non-ACS/elective patients $(22.0 \%$ vs. $13.8 \%$, respectively; odds ratio [OR] $1.8,95 \% \mathrm{CI}$ $1.3-2.5 ; p<0.001)$. This difference was primarily driven by 

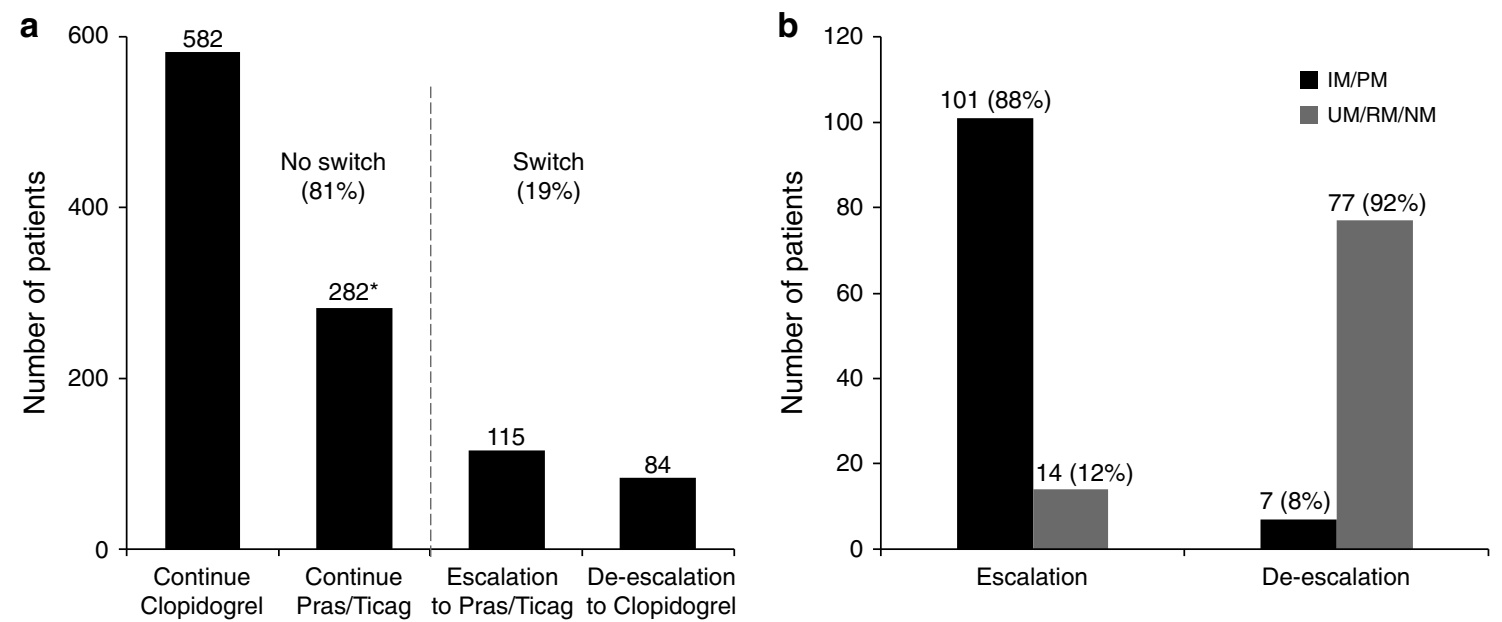

Fig. $1 \mathrm{P}_{2} \mathrm{Y}_{12}$ inhibitor maintenance therapy selection and switching by CYP2C19 status. a Maintenance therapy distribution by switch status in genotyped patients $(n=1063)$. Left panel: initiated and continued on clopidogrel or alternative therapy (i.e., not switched). ${ }^{*}$ Two switched from prasugrel to ticagrelor; one from ticagrelor to prasugrel. Right panel: switched to alternative therapy (escalation) or to clopidogrel (de-escalation). $\mathbf{b}$ Distribution of escalation and de-escalation by CYP2C19 phenotype. IM intermediate metabolizer, NM normal metabolizer, PM poor metabolizer, RM rapid metabolizer, UM ultrarapid metabolizer.

higher frequency of de-escalation to clopidogrel $(10.7 \%$ vs. $3.7 \%$, respectively, $p<0.001)$, since no difference in escalation frequency across ACS status was observed $(11.3 \%$ vs. $10.0 \%$, respectively, $p=0.505$ ) (Table S2).

Consistent with genotype-guided prescribing, escalation to prasugrel/ticagrelor occurred almost exclusively in IM/PMs (OR 22.8, 95\% CI 12.8-40.6, $p<0.001$ ), and deescalation to clopidogrel occurred primarily in UM/RM/ NMs (OR 5.4, 95\% CI 2.5-11.8, $p<0.001$ ) (Fig. 1b). Among IM/PMs initiated on clopidogrel, 101 (51.0\%) were escalated to prasugrel/ticagrelor and 97 (49.0\%) were continued on clopidogrel. Among UM/RM/NMs initiated on prasugrel/ticagrelor, $77(32.8 \%)$ were de-escalated to clopidogrel and $158(67.2 \%)$ were continued on prasugrel/ ticagrelor.

\section{Clinical outcomes}

During a median follow-up of 9.2 [4.7-11.1] months, 124 (13.4\%) experienced MACCE and 41 (4.4\%) experienced a clinically significant bleeding event. To examine the clinical impact of genotype-guided escalation and de-escalation, outcomes were evaluated following stratification by initial therapy (Fig. 2a). In patients initiated on clopidogrel (Table S3) or prasugrel/ticagrelor (Table S4), most baseline characteristics were similar across CYP2C19 phenotypemaintenance therapy groups. However, clopidogrel was more frequently used as maintenance therapy in patients with bleeding risk factors.

In patients initiated on clopidogrel, continuation of clopidogrel in IM/PMs was associated with a significantly higher risk of experiencing either a MACCE or bleeding event compared with IM/PMs escalated to alternative therapy (51.8 vs. 19.4 events per 100 patient-years, respectively; adjusted HR 2.89, 95\% CI 1.44-6.13, $p=0.003$ ) (Fig. 2b; Table 1). In contrast, no significant difference was observed in
UM/RM/NMs continued on clopidogrel compared with IM/PMs escalated to alternative therapy (adjusted HR 1.37, 95\% CI $0.76-2.70, p=0.304$ ). Differences in risk for MACCE were responsible for the observed association since IM/PMs continued on clopidogrel exhibited a significantly higher risk of MACCE compared with IM/PMs escalated to alternative therapy (adjusted HR 5.72, 95\% CI 2.41-15.81, $p<0.001$ ). In contrast, no significant differences in risk of clinically significant bleeding events were observed across groups (Table 1, Fig. S2).

In patients initiated on prasugrel/ticagrelor, de-escalation to clopidogrel in UM/RM/NMs was associated with no difference in risk of experiencing either a MACCE or bleeding event compared with those continued on alternative therapy (21.3 vs. 19.3 events per 100 patient-years, respectively; adjusted HR 1.13, 95\% CI 0.51-2.34, $p=0.751$ ). No significant difference was observed when de-escalation was compared with either IM/PMs (adjusted HR 1.35, 95\% CI $0.54-3.27, p=0.511)$ or $\mathrm{UM} / \mathrm{RM} / \mathrm{NMs}$ continued on alternative therapy (adjusted HR 0.99, 95\% CI 0.43-2.21, $p=$ 0.990) (Fig. 2c). De-escalation to clopidogrel was also associated with no difference in risk of MACCE alone compared with continued alternative therapy (adjusted HR $0.93,95 \%$ CI $0.35-2.19, p=0.878$ ). Moreover, no significant difference in risk of bleeding events was observed across groups (Table 2, Fig. S2).

Secondary analyses in patients with an ACS indication for PCI demonstrated similar results with the overall study population (Fig. S3). In ACS patients initiated on alternative therapy, the risk of experiencing either a MACCE or bleeding event (adjusted HR 1.38, 95\% CI 0.56-3.15, $p=0.461$ ) or MACCE alone (adjusted HR 1.00, 95\% CI 0.31-2.77, $p=$ 0.998 ) was not significantly different in UM/RM/NMs deescalated to clopidogrel compared with those continued on alternative therapy. 
a

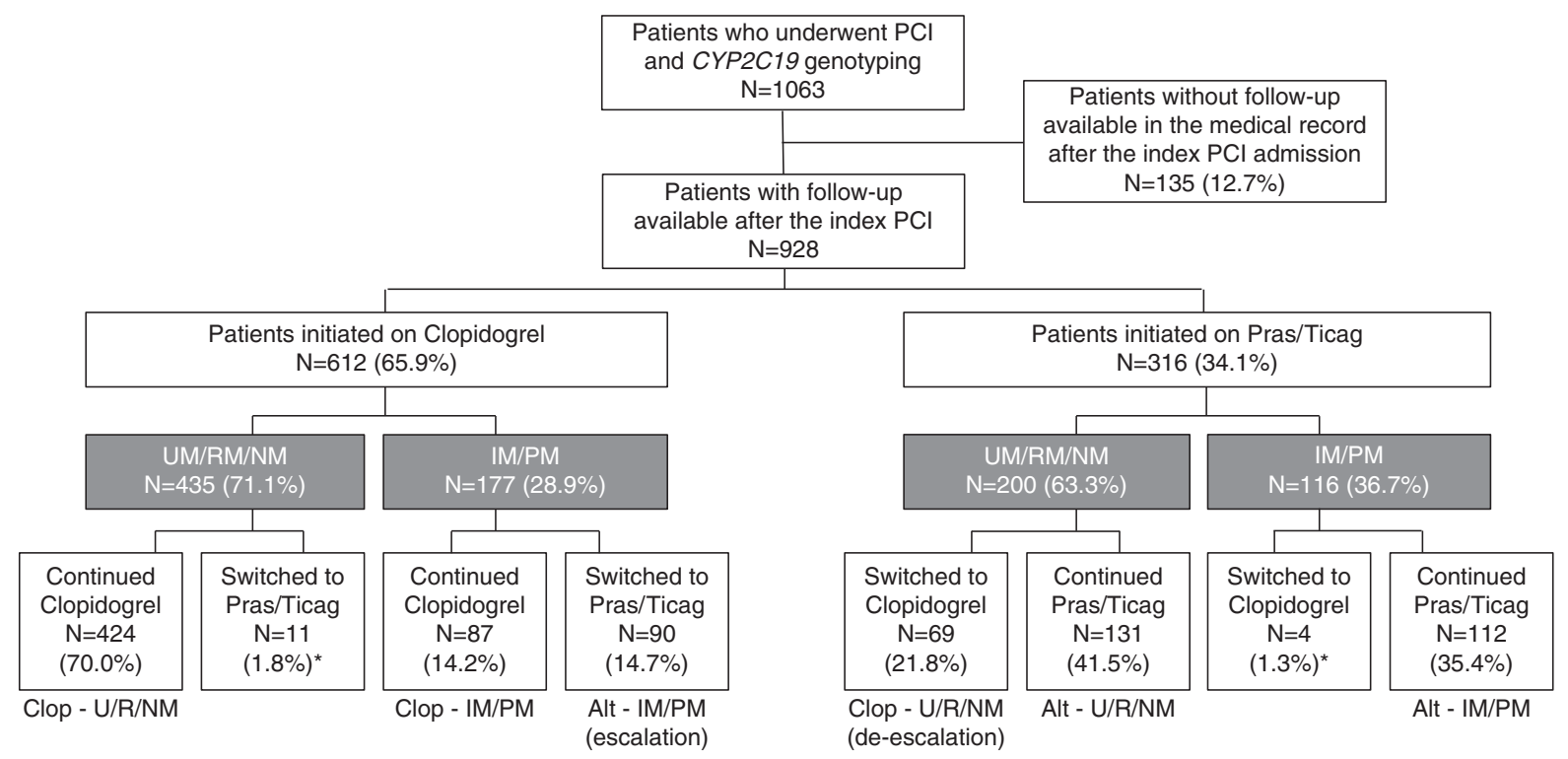

b

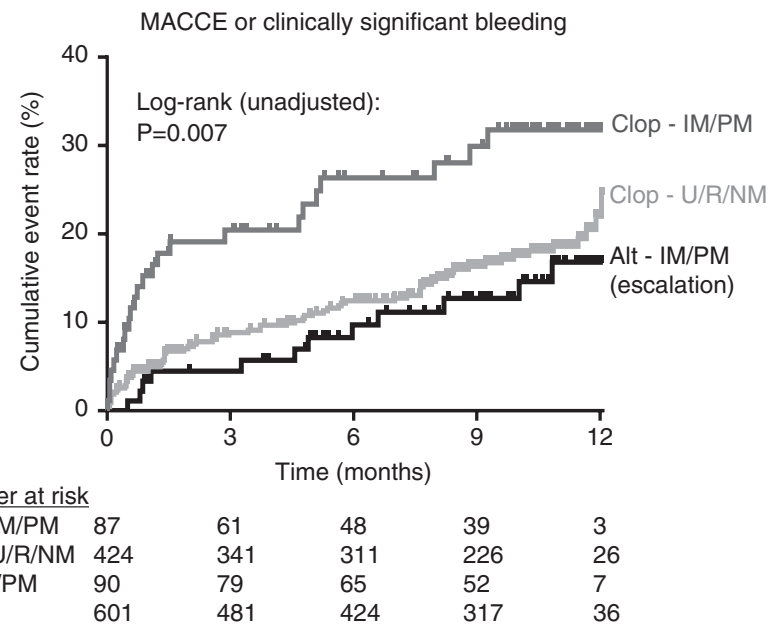

C

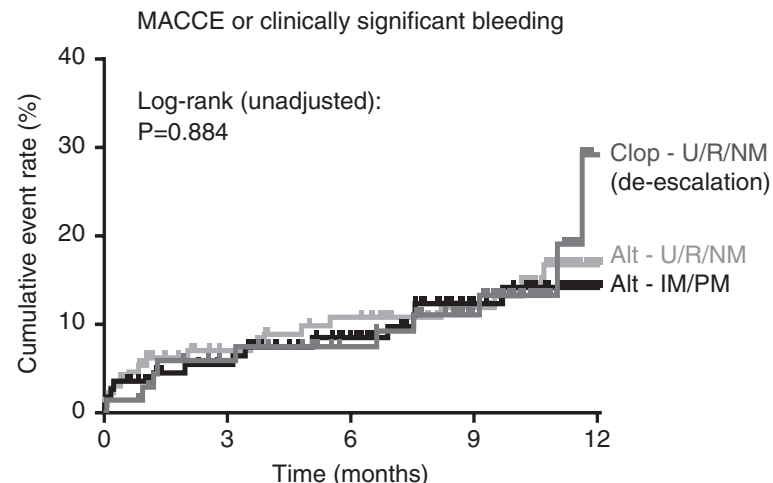

Number at risk

Clop-U/R/NM 69

Alt-U/R/NM 131

Alt-IM/PM 112

Total 312

$\begin{array}{llll}61 & 53 & 41 & 3 \\ 105 & 93 & 73 & 6 \\ 97 & 80 & 52 & 5 \\ 263 & 226 & 166 & 14\end{array}$

$\begin{array}{llll}263 & 226 & 166 & 14\end{array}$

Fig. 2 Cardiovascular or bleeding events over 12 months following percutaneous coronary intervention (PCI) by CYP2C19 status and initial and maintenance $\mathbf{P 2 Y}_{12}$ inhibitor therapy. a Study population summary by initial P2Y 12 inhibitor, CYP2C19 phenotype, and maintenance therapy. b, c Kaplan-Meier curves describing cumulative event rates for the composite of either a major adverse cardiovascular or cerebrovascular event (MACCE) or bleeding event after stratifying by initial therapy: b clopidogrel, c prasugrel/ticagrelor. Data shown across IM/PMs prescribed clopidogrel (Clop-IM/PM), IM/ PMs prescribed prasugrel/ticagrelor (Alt-IM/PM), UM/RM/NMs prescribed clopidogrel (Clop-U/R/NM), and UM/RM/NMs prescribed prasugrel/ticagrelor (AltU/R/NM). *Due to rare occurrence, UM/RM/NMs switched to prasugrel/ticagrelor and IMs switched to clopidogrel were not included in the outcome analysis. The unadjusted log-rank $P$ value is provided. IM intermediate metabolizer, $N M$ normal metabolizer, $P M$ poor metabolizer, $R M$ rapid metabolizer, UM ultrarapid metabolizer.

\section{Time to switch in $\mathrm{P}_{2} \mathrm{Y}_{12}$ inhibitor therapy}

The timing of escalation and de-escalation varied, but de-escalation to clopidogrel occurred significantly later after PCI than escalation to prasugrel/ticagrelor $(20[2-39]$ vs. 4 [2-17] days, respectively, $p=0.001)$. Accordingly, escalation occurred more frequently within 7 days $(64.3 \%$ vs. $38.1 \%, p<$ $0.001)$ and 30 days $(85.2 \%$ vs. $58.3 \%, p<0.001)$ after PCI than de-escalation, respectively. When evaluated exclusively in IM/ $\mathrm{PMs}$ and $\mathrm{UM} / \mathrm{RM} / \mathrm{NMs}$, respectively, time to de-escalation in $\mathrm{UM} / \mathrm{RM} / \mathrm{NMs}$ occurred significantly later after PCI than escalation in IM/PMs (Fig. 3).

\section{DISCUSSION}

The current study evaluated the frequency and timing of antiplatelet therapy escalation and de-escalation, and clinical outcomes in PCI patients following the implementation of a genotype-guided selection strategy in a real-world setting. Results illustrated that CYP2C19-guided escalation and de-escalation were both common, and de-escalation from prasugrel/ticagrelor to clopidogrel in UM/RM/NMs occurred significantly later after PCI than escalation from clopidogrel to prasugrel/ticagrelor in IM/PMs. In patients initiated on clopidogrel, continuation of clopidogrel in IM/PMs was 


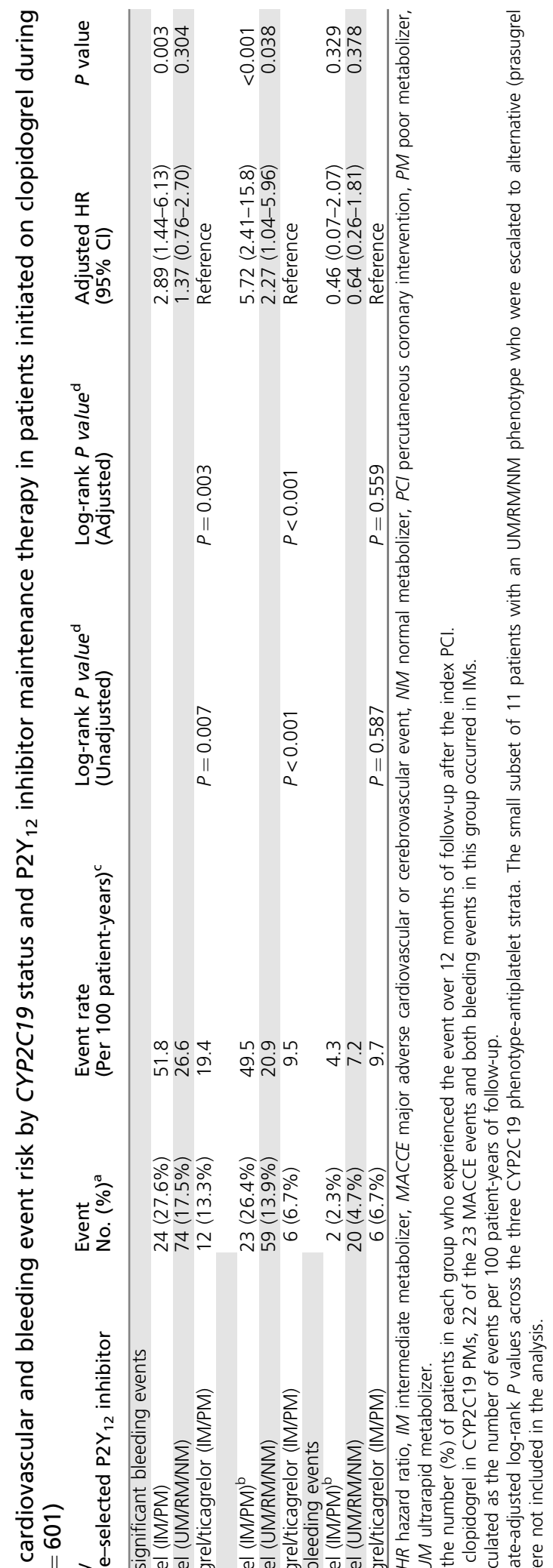

u ह

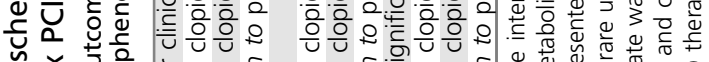

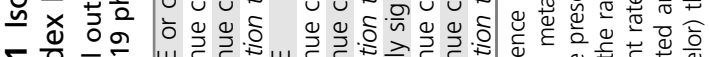

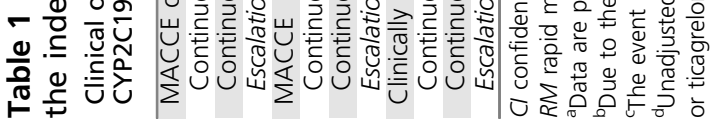

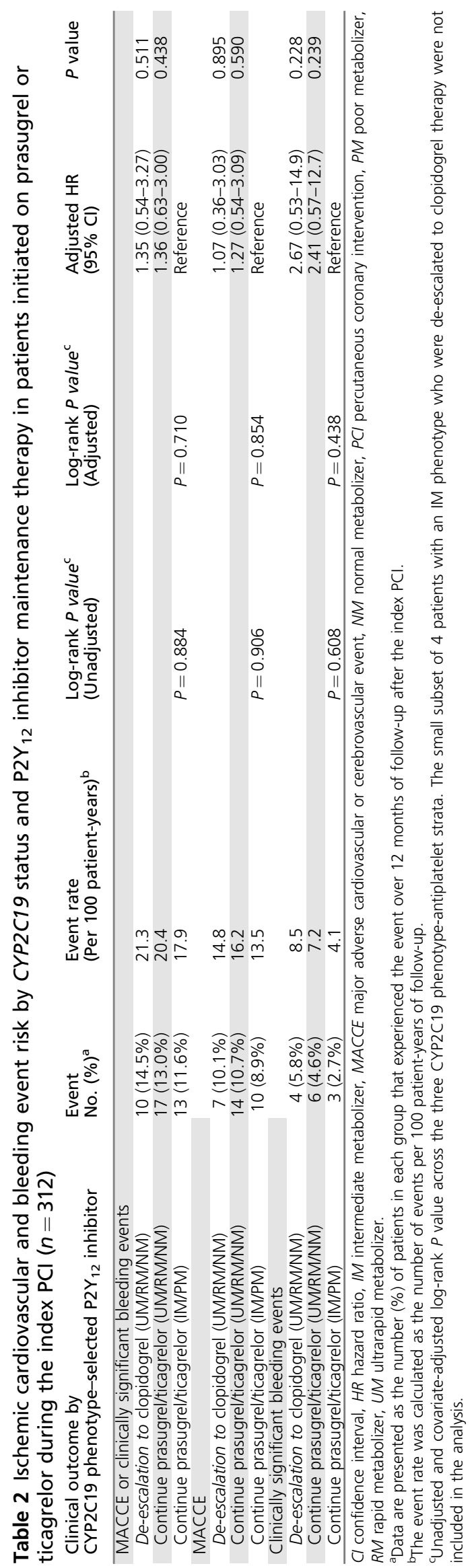


Time to Prasugrel/Ticagrelor Therapy

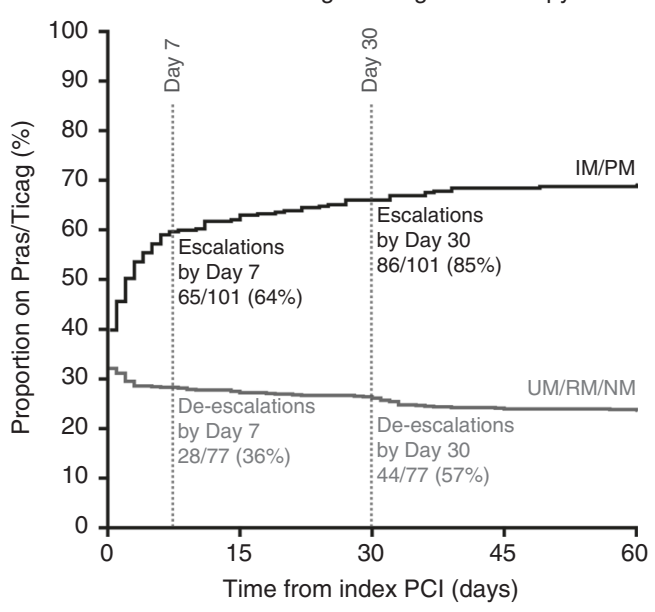

Fig. 3 Time to escalation and de-escalation of $\mathrm{P}_{2} \mathrm{Y}_{12}$ inhibitor therapy by CYP2C19 status. The cumulative frequency of initiating prasugrel or ticagrelor maintenance therapy in IM/PMs $(N=329)$ and UM/RM/NMs $(N=734)$ is presented as a function of time following the index percutaneous coronary intervention $(\mathrm{PCI})$ procedure. $I M$ intermediate metabolizer, NM normal metabolizer, PM poor metabolizer, $R M$ rapid metabolizer, UM ultrarapid metabolizer.

associated with a significantly higher risk of MACCE compared with genotype-guided escalation to alternative therapy. In patients initiated on prasugrel or ticagrelor, however, genotype-guided de-escalation to clopidogrel in $\mathrm{UM} / \mathrm{RM} / \mathrm{NMs}$ was associated with no difference in MACCE risk compared with those who continued alternative therapy. Taken together, these data illustrate that genotype-guided escalation to prasugrel or ticagrelor mitigates the risk of adverse cardiovascular outcomes conferred by clopidogrel use in CYP2C19 nonfunctional allele carriers, and that use of genotype to selectively guide de-escalation to clopidogrel in patients without a CYP2C19 nonfunctional allele appeared safe and effective.

Clopidogrel, prasugrel, and ticagrelor exhibit distinct pharmacological characteristics, and selection of the optimal antiplatelet therapy is a patient-centric, multifactorial clinical decision. Accordingly, numerous questions surrounding P2 $\mathrm{Y}_{12}$ inhibitor switching have emerged. ${ }^{11,12}$ Several prospective registry studies and post hoc observational analyses have described the frequency and clinical impact of empiric (nonguided) antiplatelet switching; ${ }^{13-18}$ however, varying definitions of switching as well as regional and temporal differences in prescribing complicate their interpretation. ${ }^{9}$ In the TRANSLATE-ACS registry, in-hospital and postdischarge switch frequencies were $12 \%$ and $8 \%$, respectively, ${ }^{14,15}$ while the ACTION-GWTG and CathPCI Registry reported inhospital switching at $6 \%$, with considerable fluctuations across the study period. ${ }^{17}$ In-hospital switches were primarily escalation to prasugrel/ticagrelor, ${ }^{14,17}$ while postdischarge switches were primarily de-escalation to clopidogrel. ${ }^{17}$ Despite the emergence of CYP2C19-guided antiplatelet selection in clinical practice, ${ }^{22}$ the frequency and timing of genotype-guided escalation and de-escalation has not been well described. In our study, the frequency of genotype-guided switching after PCI was common (19\%), and more frequent in ACS patients (22\%) compared with non-ACS/elective PCI patients (14\%). Although the frequency of switching in our study was comparable with multicenter US registries that did not include genotyping, we observed that availability of a CYP2C19 genotype result significantly increased the likelihood of a switch occurring, and that switch direction was driven by the genotype result.

CYP2C19 genotyping has been successfully implemented into clinical practice at a number of institutions..$^{22,27,31-33}$ Given the historical use of clopidogrel as first-line therapy, early genotype-guided strategies and recommendations focused on escalation of IM/PMs to alternative therapy. ${ }^{3,22}$ In our study, approximately one-half of IM/PMs initiated on clopidogrel were escalated to alternative therapy in accordance with the genotype-guided algorithm. Furthermore, our results demonstrate that failure to escalate $C Y P 2 C 19$ nonfunctional allele carriers to alternative therapy is associated with adverse cardiovascular outcomes. These data are consistent with the multicenter Implementing GeNomics In PracTicE (IGNITE) Network investigation, a multicenter randomized clinical trial in Europe, and a single-center nonrandomized trial in China, which have collectively demonstrated that genotype-guided escalation of IM/PMs to alternative therapy reduces risk of major atherothrombotic events without increasing risk of major bleeding. ${ }^{19,20,34}$

Although important, CYP2C19 genotype is one of multiple factors considered when prescribing a $\mathrm{P}_{2} \mathrm{Y}_{12}$ inhibitor. Various clinical factors, most notably risk factors for bleeding, are associated with continued clopidogrel use in IMs. ${ }^{26}$ Due to the higher risk of MACCE conferred by clopidogrel use in CYP2C19 nonfunctional allele carriers, particularly during the first 30 days after $\mathrm{PCI},{ }^{35,36}$ these results suggest that placing greater weight on an IM/PM result during the prescribing decision may be warranted. Although no difference in risk of clinically significant bleeding events was observed across groups, these results should be interpreted with caution since patients with higher underlying bleeding risk are more frequently prescribed clopidogrel. Furthermore, bleeding event rates were low and larger sample sizes are needed. The clinical benefit of genotype-guided antiplatelet therapy in patients undergoing non-ACS/elective PCI also remains unclear and requires further study. Low MACCE rates in elective PCI patients precluded investigation of outcomes in the current study. Two large randomized trials with results expected in 2020, TAILOR-PCI (NCT01742117) in elective and ACS PCI patients and POPULAR-Genetics (NCT01761786) in ST-elevation myocardial infarction (STEMI) PCI patients, are examining the clinical utility of genotype-guided antiplatelet therapy and will provide insight into these gaps in evidence. . $^{29,37}$

Efficiently and sustainably operationalizing CYP2C19 genotype-guided strategies in real-world settings can be constrained by logistical challenges. ${ }^{22}$ Barriers include onsite availability of genotype tests, turnaround time, patient 
and provider education, and the lack of clinical decision support tools to report results. Due to the higher risk of MACCE during the early post-PCI period, operational inefficiencies that delay genotype-guided escalation to alternative therapy in IM/PMs may result in negative clinical consequences. Genotype-guided de-escalation to clopidogrel in patients without a CYP2C19 nonfunctional allele after the early post-PCI period offers a practical solution to these challenges, and thus has emerged as a new strategy for genotype-guided antiplatelet therapy.

Use of prasugrel or ticagrelor early after PCI, followed by de-escalation to clopidogrel maintenance therapy, has become more common in clinical practice. ${ }^{11,12}$ This is likely due to updated clinical practice guidelines recommending preferential use of prasugrel/ticagrelor after PCI in ACS patients, ${ }^{2}$ and the higher bleeding risks, discontinuation rates, and costs associated with these agents during chronic therapy. ${ }^{10-12}$ However, there are conflicting clinical outcomes data with an empiric (nonguided) de-escalation approach, with very early de-escalation to clopidogrel after ACS increasing risk of recurrent atherothrombotic events. ${ }^{16,24}$ The TROPICAL-ACS randomized trial recently showed that a de-escalation strategy guided by platelet function testing (PFT) was noninferior to universal prasugrel treatment in the prevention of major atherothrombotic or bleeding events after ACS and PCI. ${ }^{25}$ However, PFT must be completed during treatment, which may not be feasible in a real-world setting. In the genotyping substudy, CYP2C19 nonfunctional alleles were an independent predictor of high platelet reactivity in clopidogrel-treated patients, suggesting that CYP2C19 testing might be useful in selecting patients for de-escalation. ${ }^{38}$ As genotyping can be done a priori, it may represent a more practical approach for guided de-escalation in the outpatient setting than PFT; however, the impact of using genotype to guide de-escalation on clinical outcomes had not been evaluated until the present study.

In our study, approximately one-third of UM/RM/NMs initiated on alternative therapy were de-escalated to clopidogrel. The timing of de-escalation after PCI varied across patients, and occurred on average 20 days after PCI. Most importantly, de-escalation to clopidogrel in UM/RM/NMs was associated with no significant difference in MACCE risk compared with continuation of prasugrel/ticagrelor. Moreover, no significant differences were observed in high-risk patients with an ACS indication for PCI. While these results suggest that a genotype-guided de-escalation strategy is safe and effective in a real-world setting, our sample size was limited and should be interpreted with caution until validated in larger, multicenter populations. Likewise, a randomized trial of genotype-guided de-escalation may ultimately be of value. Our results provide the foundation for these future studies.

We also observed that $\mathrm{UM} / \mathrm{RM} / \mathrm{NMs}$ initiated and continued on clopidogrel appeared to exhibit a moderately higher risk of MACCE compared with IM/PMs escalated to alternative therapy, albeit to a significantly lesser degree than
IM/PMs continued on clopidogrel. This suggests there are important gaps in evidence surrounding the key clinical and genetic factors that contribute to clopidogrel effectiveness in patients without a CYP2C19 ${ }^{\star} 2$ or $\star_{3}$ nonfunctional allele. For instance, only the ${ }^{*} 2,{ }^{*}$, and ${ }^{*} 17$ alleles were genotyped. Additional nonfunctional CYP2C19 variant alleles $\left({ }^{*} 4-{ }^{*} 8\right)$ assigned to IM/PM phenotypes, ${ }^{3}$ although rare, were not evaluated, which is a limitation. Furthermore, interindividual variation in the aspirin's antiplatelet effects may influence outcomes after PCI. ${ }^{39}$ Additional studies are needed to determine whether genetic factors beyond CYP2C19 should be used to optimize antiplatelet therapy.

It is important to acknowledge several limitations with our study. First, data collection was completed retrospectively via EHR abstraction. Thus, factors that contributed to antiplatelet selection and switching could not be conclusively determined. Second, genotype-guided therapy was not randomized and a standardized protocol for the timing of de-escalation after PCI was not employed. While the pragmatic study design minimized influence on the clinical practice, we cannot attribute cause and effect or exclude the influence of bias to the observed associations between genotype-guided escalation and de-escalation and clinical outcomes. Although covariate-adjusted and stratified analyses were conducted to lessen the potential confounding effects related to baseline differences observed across CYP2C19 phenotype-antiplatelet therapy groups, such as diabetes and bleeding risk factors, socioeconomic factors were not evaluated and residual confounding may remain. Thus, the magnitude of the observed associations should be interpreted with caution. Lastly, these data reflect the experience with genotype-guided therapy at a single center, and may not be generalizable to other settings and populations. For instance, the low utilization of ticagrelor in our cohort does not reflect contemporary prescribing patterns of increased ticagrelor use. ${ }^{9}$ Future studies in more diverse populations are warranted.

In summary, use of a CYP2C19-guided antiplatelet therapy strategy after PCI in a real-world setting commonly necessitates both escalation of nonfunctional allele carriers to alternative therapy, and de-escalation of patients without a nonfunctional allele to clopidogrel. Genotype-guided escalation to prasugrel/ticagrelor mitigates the risk of adverse cardiovascular outcomes conferred by continued clopidogrel use in CYP2C19 nonfunctional allele carriers. In addition, early use of prasugrel/ticagrelor followed by genotype-guided de-escalation to clopidogrel maintenance therapy in those without a CYP2C19 nonfunctional allele appears to be a safe and effective strategy that warrants further study.

\section{SUPPLEMENTARY INFORMATION}

The online version of this article (https://doi.org/10.1038/s41436019-0611-1) contains supplementary material, which is available to authorized users. 


\section{ACKNOWLEDGEMENTS}

The authors gratefully acknowledge the UNC Cardiac Catheterization Laboratory and the UNC Molecular Genetics Laboratory staff for their important contributions. We also thank our partners in the IGNITE Network, a consortium of genomic medicine pilot demonstration projects funded and guided by the National Human Genome Research Institute (NHGRI) (https://ignitegenomics.org/), for their valuable contributions. The project described was supported by the National Center for Advancing Translational Sciences (NCATS), National Institutes of Health, through grant award number UL1TR002489. The contents are solely the responsibility of the authors and do not necessarily represent the official views of the $\mathrm{NHGRI}$ or $\mathrm{NIH}$.

\section{DISCLOSURE}

The authors declare no conflicts of interest.

Publisher's note: Springer Nature remains neutral with regard to jurisdictional claims in published maps and institutional affiliations.

\section{REFERENCES}

1. Benjamin EJ, Virani SS, Callaway CW, et al. Heart disease and stroke statistics -2018 update: a report from the American Heart Association. Circulation. 2018:137:E67-E492.

2. Levine GN, Bates ER, Bittl JA, et al. 2016 ACC/AHA guideline focused update on duration of dual antiplatelet therapy in patients with coronary artery disease. J Am Coll Cardiol. 2016;68:1082 LP-1081115.

3. Scott SA, Sangkuhl K, Stein CM, et al. Clinical Pharmacogenetics Implementation Consortium guidelines for CYP2C19 genotype and clopidogrel therapy: 2013 update. Clin Pharmacol Ther. 2013;94:317-323.

4. Mega JL, Simon T, Collet J-P, et al. Reduced-function CYP2C19 genotype and risk of adverse clinical outcomes among patients treated with clopidogrel predominantly for PCl: a meta-analysis. JAMA. 2010;304:1821-1830.

5. Wiviott SD, Braunwald E, McCabe $\mathrm{CH}$, et al. Prasugrel versus clopidogrel in patients with acute coronary syndromes. N Engl J Med. 2007;357:2001-2015.

6. Wallentin L, Becker RC, Budaj A, et al. Ticagrelor versus clopidogrel in patients with acute coronary syndromes. N Engl J Med. 2009; 361:1045-1057.

7. Mega JL, Close SL, Wiviott SD, et al. Cytochrome P450 genetic polymorphisms and the response to prasugrel: relationship to pharmacokinetic, pharmacodynamic, and clinical outcomes. Circulation. 2009;119:2553-2560.

8. Wallentin L, James S, Storey RF, et al. Effect of CYP2C19 and ABCB1 single nucleotide polymorphisms on outcomes of treatment with ticagrelor versus clopidogrel for acute coronary syndromes: a genetic substudy of the PLATO trial. Lancet. 2010;376:1320-1328.

9. Dayoub EJ, Seigerman M, Tuteja S, et al. Trends in platelet adenosine diphosphate P2Y12 receptor inhibitor use and adherence among antiplatelet-naive patients after percutaneous coronary intervention, 2008-16. JAMA Intern Med. 2018;178:943-950.

10. Zanchin T, Temperli F, Karagiannis A, et al. Frequency, reasons, and impact of premature ticagrelor discontinuation in patients undergoing coronary revascularization in routine clinical practice: results from the Bern Percutaneous Coronary Intervention Registry. Circ Cardiovasc Interv. 2018;11:e006132.

11. Angiolillo DJ, Rollini F, Storey RF, et al. International expert consensus on switching platelet P2Y12 receptor-inhibiting therapies. Circulation. 2017;136:1955-1975.

12. Rollini F, Franchi F, Angiolillo DJ. Switching P2Y12-receptor inhibitors in patients with coronary artery disease. Nat Rev Cardiol. 2016;13:11-27.

13. Alexopoulos D, Xanthopoulou I, Deftereos $S$, et al. In-hospital switching of oral P2Y12 inhibitor treatment in patients with acute coronary syndrome undergoing percutaneous coronary intervention: prevalence, predictors and short-term outcome. Am Heart J. 2014;167:68-76.e2.
14. Bagai A, Peterson ED, Honeycutt E, et al. In-hospital switching between adenosine diphosphate receptor inhibitors in patients with acute myocardial infarction treated with percutaneous coronary intervention: insights into contemporary practice from the TRANSLATE-ACS study. Eur Hear J Acute Cardiovasc Care. 2014;4:499-508.

15. Zettler ME, Peterson ED, McCoy LA, et al. Switching of adenosine diphosphate receptor inhibitor after hospital discharge among myocardial infarction patients: insights from the Treatment with Adenosine Diphosphate Receptor Inhibitors: Longitudinal Assessment of Treatment Patterns and Events after Acute Coronary Syndrome (TRANSLATE-ACS) observational study. Am Heart J. 2017;183:62-68.

16. De Luca L, D'Ascenzo F, Musumeci G, et al. Incidence and outcome of switching of oral platelet P2Y12 receptor inhibitors in patients with acute coronary syndromes undergoing percutaneous coronary intervention: the SCOPE registry. Eurolntervention. 2017;13:459-466.

17. Bagai A, Wang $Y$, Wang TY, et al. In-hospital switching between clopidogrel and prasugrel among patients with acute myocardial infarction treated with percutaneous coronary intervention: insights into contemporary practice from the national cardiovascular data registry. Circ Cardiovasc Interv. 2014; 7:585-593.

18. Almendro-Delia M, Blanco Ponce E, Gomez-Dominguez R, et al. Safety and efficacy of in-hospital clopidogrel-to-prasugrel switching in patients with acute coronary syndrome. An analysis from the "real world". J Thromb Thrombolysis. 2015;39:499-507.

19. Cavallari $\mathrm{LH}$, Lee $\mathrm{CR}$, Beitelshees $\mathrm{AL}$, et al. Multisite investigation of outcomes with implementation of CYP2C19 genotype-guided antiplatelet therapy after percutaneous coronary intervention. JACC Cardiovasc Interv. 2018;11:181-191.

20. Notarangelo FM, Maglietta G, Bevilacqua $P$, et al. Pharmacogenomic approach to selecting antiplatelet therapy in patients with acute coronary syndromes: the PHARMCLO Trial. J Am Coll Cardiol. 2018;71:1869-1877.

21. Klein MD, Williams AK, Lee CR, Stouffer GA. Clinical utility of CYP2C19 genotyping to guide antiplatelet therapy in patients with an acute coronary syndrome or undergoing percutaneous coronary intervention. Arterioscler Thromb Vasc Biol. 2019;39:647-652.

22. Empey $\mathrm{PE}$, Stevenson $\mathrm{JM}$, Tuteja $\mathrm{S}$, et al. Multisite investigation of strategies for the implementation of CYP2C19 genotype-guided antiplatelet therapy. Clin Pharmacol Ther. 2018;104:664-674.

23. Alexopoulos D, Lianos I, Vlachakis P, Sfantou D, Dragona VM, Varlamos C. De-escalation of treatment with oral P2Y12 receptor inhibitors: current status and perspectives. J Cardiovasc Pharmacol Ther. 2019;214:304-314.

24. Cuisset T, Deharo P, Quilici J, et al. Benefit of switching dual antiplatelet therapy after acute coronary syndrome: the TOPIC (timing of platelet inhibition after acute coronary syndrome) randomized study. Eur Heart J. 2017;38:3070-3078.

25. Sibbing D, Aradi DD, Jacobshagen $C$, et al. Guided de-escalation of antiplatelet treatment in patients with acute coronary syndrome undergoing percutaneous coronary intervention (TROPICAL-ACS): a randomised, open-label, multicentre trial. Lancet. 2017;390:1747-1757.

26. Lee CR, Sriramoju VB, Cervantes A, et al. Clinical outcomes and sustainability of using CYP2C19 genotype-guided antiplatelet therapy after percutaneous coronary intervention. Circ Genomic Precis Med. 2018; 11:e002069.

27. Lee $J A$, Lee $C R$, Reed $B N$, et al. Implementation and evaluation of a CYP2C 19 genotype-guided antiplatelet therapy algorithm in high-risk coronary artery disease patients. Pharmacogenomics. 2015;16:303-313.

28. Caudle KE, Dunnenberger HM, Freimuth RR, et al. Standardizing terms for clinical pharmacogenetic test results: consensus terms from the Clinical Pharmacogenetics Implementation Consortium (CPIC). Genet Med. 2017;19:215-223.

29. Bergmeijer TO, Janssen PWA, Schipper JC, et al. CYP2C19 genotypeguided antiplatelet therapy in ST-segment elevation myocardial infarction patients - rationale and design of the Patient Outcome after primary PCI (POPular) Genetics study. Am Heart J. 2014;168:16-22.e1.

30. Mehran R, Rao SV, Bhatt DL, et al. Standardized bleeding definitions for cardiovascular clinical trials: a consensus report from the Bleeding Academic Research Consortium. Circulation. 2011;123:2736-2747.

31. Weitzel KW, Elsey AR, Langaee TY, et al. Clinical pharmacogenetics implementation: approaches, successes, and challenges. Am J Med Genet C Semin Med Genet. 2014;166:56-67.

32. Peterson JF, Field JR, Unertl KM, et al. Physician response to implementation of genotype-tailored antiplatelet therapy. Clin Pharmacol Ther. 2016;100: 67-74. 
33. Cavallari LH, Franchi F, Rollini F, et al. Clinical implementation of rapid CYP2C19 genotyping to guide antiplatelet therapy after percutaneous coronary intervention. J Transl Med. 2018;16:92.

34. Shen D-L, Wang B, Bai J, et al. Clinical value of CYP2C19 genetic testing for guiding the antiplatelet therapy in a Chinese population. J Cardiovasc Pharmacol. 2016;67:232-236.

35. Williams AK, Klein MD, Martin J, et al. CYP2C19 genotype-guided antiplatelet therapy and 30-day outcomes after percutaneous coronary intervention. Circ Genomic Precis Med. 2019;12:e002441.

36. Borse MS, Dong OM, Polasek MJ, Farley JF, Stouffer GA, Lee CR. CYP2C19-guided antiplatelet therapy: a cost-effectiveness analysis of 30-day and 1-year outcomes following percutaneous coronary intervention. Pharmacogenomics. 2017;18:1155-1166.

37. Pereira NL, Rihal CS, So DYF, et al. Clopidogrel pharmacogenetics. Circ Cardiovasc Interv. 2019;12:e007811.

38. Gross L, Trenk D, Jacobshagen C, et al. Genotype-phenotype association and impact on outcomes following guided de-escalation of anti-platelet treatment in acute coronary syndrome patients: the TROPICAL-ACS genotyping substudy. Thromb Haemost. 2018;118:1656-1667.

39. Tantry US, Gesheff M, Liu F, Bliden KP, Gurbel PA. Resistance to antiplatelet drugs: what progress has been made? Expert Opin Pharmacother. 2014; $15: 2553-2564$. 